\title{
Niveles de evidencia y grados de recomendación (I). Hacia la perspectiva GRADE
}

\author{
R. Aguilera Eguía ${ }^{1}$, E. Zafra Santos², C. Rojas Sepúlveda³, G. Aguayo Alcayaga ${ }^{3}$, D. Sánchez León ${ }^{3}$ \\ y T. Aguilera Eguía ${ }^{4}$ \\ ${ }^{I}$ Musculoskeletal Group Cochrane Colaboration. Member of the Grade Working Group. Member of the \\ Cochrane Trainer's Network. Colaboración Cochrane Iberoamericana. Red Chilena de Salud Basada en \\ Evidencia. ${ }^{2}$ Escuela de Kinesiología. Universidad Santo Tomás. ${ }^{3}$ Licenciado en Kinesiología. ${ }^{4}$ Pedagogía en \\ Inglés. Universidad Alberto Hurtado. Chile
}

Aguilera Eguía R, Zafra Santos E, Rojas Sepúlveda C, Aguayo Alcayaga G, Sánchez León D, Aguilera Eguía T. Niveles de evidencia y grados de recomendación (I). Hacia la perspectiva GRADE. Rev Soc Esp Dolor 2014; 21(2): 92-96.

\begin{abstract}
Nowadays, it is being important to take clinical decisions based on the best levels of evidence and grades of recommendation because both concepts are a fundamental pillar of evidence-based practice. Currently we can count over a hundred tools, 19 systems to assess the quality and 7 to grade the recommendations, the scales can use letters (A, B, C, etc.), numbers (I, II, III) or a combination of both (Ia, IIb, Ila, etc.) but the way to express the levels of evidence and grades of recommendation is quite unequal among different scales, showing differences in the criteria of graduation, reproducibility, and interpretation of the grades of recommendation, that can confuse and increase the uncertainty when making a clinical decision.
\end{abstract}

The purpose of our work is to show the initiatory GRADE.

Key words: Systematic reviews. Clinical practice guidelines. Health technology assessment. Quality of evidence. Strength of recommendations.

\section{RESUMEN}

Hoy en día, cada vez tiene más peso el tomar una decisión clínica fundamentada en los mejores niveles de evidencia y grados de recomendación, debido a que ambos conceptos constituyen un pilar fundamental de la práctica basada en evi-

Recibido: 01-03-13.

Aceptado: 15-06-13 dencia. Actualmente podemos contabilizar más de cien herramientas, 19 sistemas para evaluar la calidad y 7 para graduar las recomendaciones, las escalas pueden utilizar letras (A, B, C, etc.), números (I, II, III) o una combinación de ambos (Ia, IIb, IIa, etc.). Sin embargo, la forma de expresar los niveles de evidencia y grados de recomendación es totalmente dispar entre las distintas escalas, presentando diferencias en los criterios de graduación, reproducibilidad e interpretación de los grados de recomendación, lo que puede confundir e incrementar la incertidumbre en el momento de tomar una decisión clínica.

El propósito de nuestro trabajo es dar a conocer la iniciativa GRADE.

Palabras clave: Revisiones sistemáticas. Guía de práctica clínica. Valoración de tecnologías en salud. Calidad de la evidencia. Fuerza de recomendación.

\section{INTRODUCCIÓN}

Esta última década se ha caracterizado por un incremento enorme y exponencial de la cantidad de información biomédica publicada, lo cual puede ser beneficioso o llegar a convertirse en un gran problema para los clínicos.

Es por este motivo que surge una nueva corriente denominada "Medicina Basada en Evidencia", la cual nace como un movimiento destinado a llenar la tradicional brecha existente entre la "práctica clínica y la investigación", promoviendo el cambio desde la toma de decisiones clínicas "informadas" para ser aplicadas en un paciente "particular".

Es por esto que hoy en día cada vez tiene más peso entre los profesionales de la salud tomar una decisión clínica fundamentada en los mejores niveles de evidencia; esto implica hasta qué punto los clínicos pueden confiar en que 
la estimación de un efecto es la adecuada para apoyar una recomendación; esto generalmente está asociado con el diseño del estudio (descriptivos o analíticos, observacionales o experimentales). También debemos recordar que cada diseño presenta diferentes características y que "no" todas las preguntas clínicas se deben responden con un mismo diseño metodológico - estudios clínicos aleatorizados (ECA) son el "patrón de oro" para responder a preguntas sobre terapia-. En este sentido, el nivel de evidencia que presenta un ECA es mayor que un estudio observacional.

La fuerza de recomendación nos indica hasta qué punto podemos confiar en si poner en práctica la recomendación nos llevará a producir más beneficios que riesgos.

El decidir si una intervención resulta "adecuada" para un paciente determinado equivale a establecer si existe un grado razonable de certeza de que el balance entre los beneficios, los efectos adversos y los costes de dichos resultados son lo suficientemente favorables como para que merezca la pena su aplicación.

En la toma de decisión clínica existen conceptos relevantes como es el caso de calidad (nivel) de la evidencia y fuerza (grado) de recomendación; ambos constituyen un pilar fundamental de la práctica basada en evidencia en su intento de "estandarizar" y proporcionar a los clínicos reglas para analizar los artículos científicos, determinar su validez y considerar su utilidad clínica (balance entre los riesgos y los beneficios).

Los conceptos mencionados anteriormente, si bien es cierto que se relacionan y complementan entre sí, se ocupan de aspectos totalmente distintos, aunque la fuerza de una recomendación se apoya en la calidad de la evidencia en la cual se sustenta. Esto no parece ser suficiente debido a que la magnitud del efecto sobre un outcome (resultado de interés) podría presentar poca precisión o ser irrelevante desde de punto de vista clínico (outcome intermedio).

Dentro de los elementos "relevantes" que debemos considerar en el momento de tomar una decisión clínica y así poder "recomendar" con "seguridad" la utilización de una intervención, hay que poner en una balanza los "posibles beneficios" versus los "posibles riesgos"; este punto es clave debido a que considera no sólo los posibles efectos adversos que pudiera presentar el fármaco administrado, sino que también incluye la magnitud del efecto, el daño, el coste, los valores y las preferencias de los pacientes.

El primer intento confiable para introducir "rigor" y "transparencia" en la jerarquización de la evidencia fue realizado hace más de 30 años por la Canadian Task Force on Preventive Health Care (CTFPHC) (1), adaptado posteriormente por la United State Preventive Services Task Force (USPSTF) (2); desde entonces numerosas organizaciones e instituciones, entre las que destacan el Centre for Evidence-Based Medicine (CEBM) de la Oxford (3), el Scottish Intercollegiate Guidelines Network (SIGN) (4), el National
Institute for Health and Clinical Excellence (NICE) (5), Van Tulder (6) y actualmente Grade Working Group (GRADE) $(7,8)$. Cada una de las instituciones mencionadas ha ido desarrollando sus propios sistemas jerárquicos.

Actualmente podemos contabilizar más de cien herramientas, 19 sistemas para evaluar la calidad y 7 para graduar las recomendaciones (8).

Las escalas pueden utilizar letras (A, B, C, etc.), números (I, II, III) o una combinación de ambos (Ia, IIb, IIa, etc.); a modo de ejemplo, si evaluamos el nivel de evidencia y el grado de recomendación de un ensayo clínico aleatorizado (9) (elegido azarosamente para el ejemplo) con las escalas mencionadas anteriormente encontraríamos lo siguiente: según la valoración del CTFPHC presenta un nivel de evidencia y grado de recomendación (IA); para SIGN (I++A); Oxford (Ib-A); USPSTF (moderada A); NICE (I+A); Van Tulder (moderada evidencia); GRADE (very low). Sin embargo, la forma de expresar los niveles de evidencia y grados de recomendación es totalmente dispar entre las escalas citadas (aun evaluando el mismo estudio y diseño metodológico), presentando diferencias en los criterios de graduación, reproducibilidad e interpretación de los grados de recomendación, lo que puede confundir e incrementar la incertidumbre en el momento de tomar una decisión clínica.

En el año 2000, con el objetivo de abordar las deficiencias de los actuales sistemas de clasificación, un grupo internacional de clínicos, y en su mayoría metodólogos, crearon la iniciativa GRADE (The Grading of Recommendations Assessment Development and Evaluation/Clasificación de la Evaluación, Desarrollo y Valoración de las Recomendaciones) y han elaborado una clasificación que presenta un enfoque sistemático y explícito a partir de la definición de una serie de criterios necesarios para hacer juicios sobre la calidad de la evidencia y la fuerza de la recomendación.

El sistema GRADE basa su decisión en los siguientes puntos:

1. Diseño del estudio: el diseño del estudio se refiere al tipo de diseño metodológico, que en términos generales se clasifica en observacional o ensayo aleatorizado.

2. Calidad del estudio: la calidad del estudio hace referencia a los métodos utilizados y a su realización. En los ensayos aleatorizados hay que tener en cuenta el ocultamiento de la asignación, el cegamiento y el seguimiento, etc.

3. Consistencia: la consistencia se refiere a la similitud en las estimaciones del efecto entre los estudios. Diferencias entre la dirección del efecto, su magnitud e importancia ayudan a determinar si hay una inconsistencia importante.

4. Evidencia directa o indirecta: la evidencia directa o indirecta indica hasta qué punto los sujetos, las inter- 
venciones y las medidas de resultado de los estudios son similares a las de nuestro interés.

Actualmente el grupo de trabajo GRADE está compuesto por 161 profesionales del área de la salud y metodólogos distribuidos por todo el mundo (Fig. 1).

Hoy en día un gran número de instituciones utilizan y apoyan la perspectiva del grupo de trabajo GRADE, entre ellas encontramos a la Organización Mundial de la Salud (OMS), la Colaboración Cochrane, el British Medical Journal (BMJ) y Clinical Evidence, entre otras (Tabla I).

\section{CONCLUSIÓN}

Con el transcurso de los años, diferentes organizaciones de ámbito mundial han intentado estandarizar la clasificación de la evidencia y la fuerza de recomendación con un solo objetivo: equilibrar, sencillez, dar claridad, transparencia y disminuir la incertidumbre en el momento de tomar una decisión clínica. Por tal motivo un grupo de clínicos y metodólogos crean la iniciativa GRADE. Este enfoque nace para desarrollar un método común y razonable para graduar la calidad de la evidencia y la fuerza de recomendación.

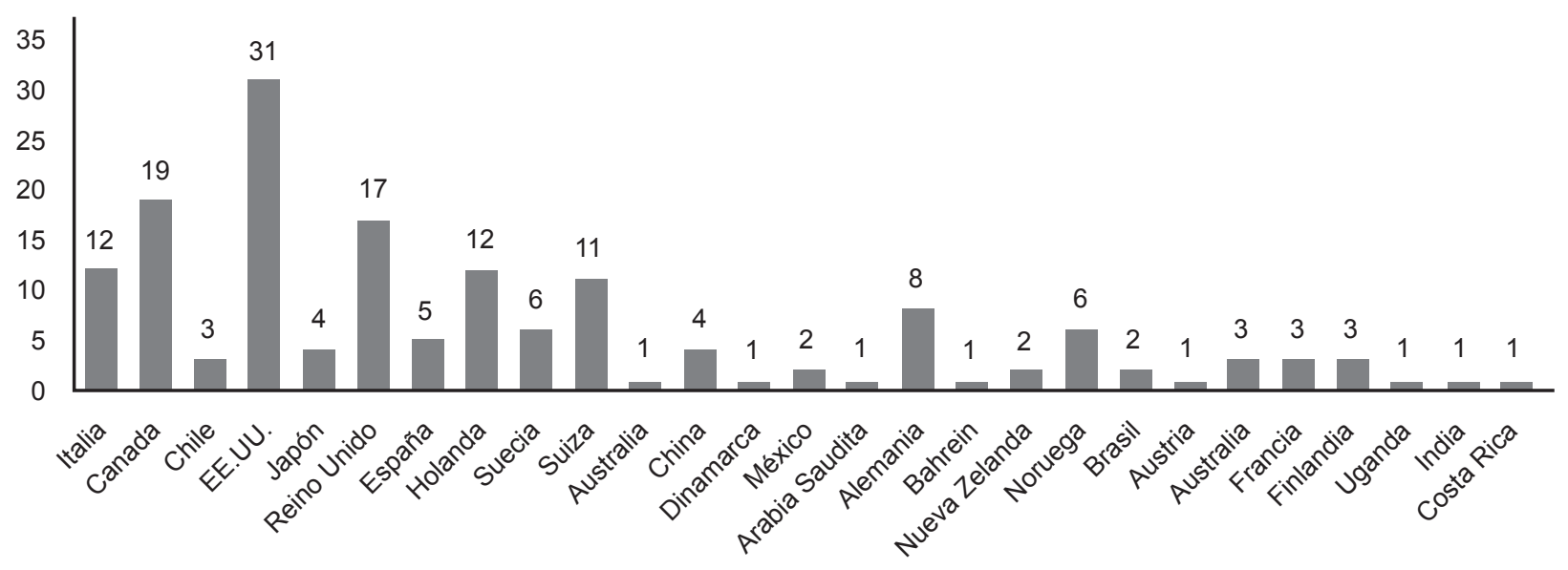

Fig. 1. Equipos de trabajo GRADE distribuidos por el mundo.

TABLA I. ORGANIZACIONES QUE UTILIZAN O APOYAN GRADE

\begin{tabular}{lcc}
\hline \multicolumn{1}{c}{ Organización } & Página web & País \\
\hline World Health Organization & www.whqlibdoc.who.int & Internacional \\
Endocrine Society & www.endo-society.org & EE.UU. \\
American College of Chest Physicians & www.chestnet.org & EE.UU. \\
UpToDate - Putting Clinical Information Into Practice & www.uptodate.com & EE.UU. \\
Agenzia sanitaria regionale & asr.regione.emilia-romagna.it & Italia \\
Ministry of Health and Long-Term Care & www.health.gov.on.ca & Canadá \\
$\begin{array}{l}\text { Surviving Sepsis } \\
\text { Ärztliches Zentrum für Qualität in der Medizin }\end{array}$ & www.survivingsepsis.org & Internacional \\
American Thoracic Society & www.aezq.de & Alemania \\
American College of Physicians & www.thoracic.org & EE.UU. \\
The Cochrane Collaboration & www.acponline.org & EE.UU. \\
Kidney Disease: Improving Global Outcome & www.cochrane.org & Internacional \\
European Society of Thoracic Surgeons & www.kdigo.org & Internacional \\
British Medical Journal & www.ests.org & Internacional \\
Journal of Infection in Developing Countries & www.bmj.com & Reino Unido \\
\hline
\end{tabular}


TABLA I. ORGANIZACIONES QUE UTILIZAN O APOYAN GRADE (CONT.)

\begin{tabular}{|c|c|c|}
\hline Organización & Página web & País \\
\hline Agency for Healthcare Research and Quality (AHRQ) & www.ahrq.gov & EE.UU. \\
\hline Society of Critical Care Medicine (SCCM) & www.sccm.org & EE.UU. \\
\hline National Institute for Clinical Excellence (NICE) & www.nice.org.uk & Reino Unido \\
\hline Norwegian Knowledge Centre for the Health Services & www.kunnskapssenteret.no & Noruega \\
\hline $\begin{array}{l}\text { The University of Pennsylvania Health System Center } \\
\text { for Evidence-based Practice }\end{array}$ & www.uphs.upenn.edu & EE.UU. \\
\hline $\begin{array}{l}\text { German Center for Evidence-based Nursing "sapere } \\
\text { aude" }\end{array}$ & www.medizin.uni-halle.de & Alemania \\
\hline Evidence-based Nursing Südtirol & www.provincia.bz.it & Italia \\
\hline Society for Vascular Surgery & www.vascularweb.org & EE.UU. \\
\hline BMJ Clinical Evidence & www.clinicalevidence.com & Reino Unido \\
\hline EBM Guidelines & www.onlinelibrary.wiley.com & Finlandia \\
\hline Polish Institute for EBM & ebm.org.pl & Polonia \\
\hline European Respiratory Society (ERS) & ersnet.org & Europa \\
\hline Japanese Society for Temporomandibular Joint & kokuhoken.net & Japón \\
\hline National Board of Health and Welfare & www.socialstyrelsen.se & Suecia \\
\hline $\begin{array}{l}\text { COMPUS at The Canadian Agency for Drugs and } \\
\text { Technologies in Health (CADTH) }\end{array}$ & cadth.ca & Canadá \\
\hline Infectious Diseases Society of America & www.idsociety.org & EE.UU. \\
\hline Spanish Society for Family and Community Medicine & www.semfyc.es & España \\
\hline $\begin{array}{l}\text { Emergency Medical Services for Children National } \\
\text { Resource Center }\end{array}$ & www.childrensnational.org & EE.UU. \\
\hline $\begin{array}{l}\text { SBU - The Swedish Council on Technology } \\
\text { Assessment in Health Care }\end{array}$ & www.sbu.se & Suecia \\
\hline $\begin{array}{l}\text { The Scottish Intercollegiate Guidelines Network } \\
\text { (SIGN) }\end{array}$ & www.sign.ac.uk & Reino Unido \\
\hline Evidence-Based Tuberculosis Diagnosis & tbevidence.org & Canadá \\
\hline $\begin{array}{l}\text { National \& Gulf Center for Evidence Based Health } \\
\text { Practice (NGCEBHP) }\end{array}$ & eapps.ngha.med.sa & Arabia Saudita \\
\hline American Society for Gastrointestinal Endoscopy & www.asge.org & EE.UU. \\
\hline European Association for the Study of the Liver & www.easl.eu & Europa \\
\hline $\begin{array}{l}\text { CDC's Healthcare Infection Control Practices Advisory } \\
\text { Committee (HICPAC) }\end{array}$ & www.cdc.gov & EE.UU. \\
\hline Finnish Office for Health Technology Assessment & finohta.stakes.fi & Finlandia \\
\hline NHS Quality Improvement Scotland & www.healthcareimprovementscotland.org & Reino Unido \\
\hline $\begin{array}{l}\text { The American Association for the Study of Liver } \\
\text { Diseases }\end{array}$ & www.aasld.org & EE.UU. \\
\hline The Canadian Cardiovascular Society & www.ccs.ca & Canadá \\
\hline The World Allergy Organization (WAO) & www.worldallergy.org & Internacional \\
\hline Kaiser Permanente & healthy.kaiserpermanente.org & EE.UU. \\
\hline $\begin{array}{l}\text { The European Monitoring Centre for Drugs and Drug } \\
\text { Addiction (EMCDDA) }\end{array}$ & www.emcdda.europa.eu & Europa \\
\hline
\end{tabular}


TABLA I. ORGANIZACIONES QUE UTILIZAN O APOYAN GRADE (CONT.)

\begin{tabular}{|c|c|c|}
\hline Organización & Página web & País \\
\hline $\begin{array}{l}\text { World Interactive Network Focused On Critical } \\
\text { UltraSound }\end{array}$ & www.winfocus.org & Internacional \\
\hline Critical Ultrasound Journal & www.springer.com & Italia \\
\hline $\begin{array}{l}\text { American Society for Colposcopy and Cervical } \\
\text { Pathology }\end{array}$ & www.asccp.org & EE.UU. \\
\hline The Dutch Institute for Healthcare Improvement CBO & www.cbo.nl & Holanda \\
\hline Kleijnen Systematic Reviews Ltd & www.systematic-reviews.com & Reino Unido \\
\hline American Gastroenterological Association & www.gastro.org & EE.UU. \\
\hline Ludwig Boltzmann Institut & hta.lbg.ac.at & Austria \\
\hline Canadian Task Force on Preventive Health Care & canadiantaskforce.ca & Canadá \\
\hline Canadian Society of Nephrology & www.csnscn.ca/en/ & Canadá \\
\hline The National Kidney Foundation/KDOQI & www.kidney.org & EE.UU. \\
\hline ICSI - Institute for Clinical Systems Improvement & www.icsi.org & EE.UU. \\
\hline $\begin{array}{l}\text { World Society of the Abdominal Compartment } \\
\text { Syndrome (WSACS) }\end{array}$ & www.wsacs.org & Internacional \\
\hline $\begin{array}{l}\text { CDC's Advisory Committee on Immunization Practices } \\
\text { (ACIP) }\end{array}$ & www.cdc.gov & EE.UU. \\
\hline The American Society of Colon and Rectal Surgeons & www.fascrs.org & EE.UU. \\
\hline The American Academy of Sleep Medicine & www.aasmnet.org & EE.UU. \\
\hline The Belgian Health Care Knowledge Centre (KCE) & kce.fgov.be & Bélgica \\
\hline Robert Koch Institute & www.rki.de & Alemania \\
\hline $\begin{array}{l}\text { Oficina de Evaluación de Medicamentos (Servicio } \\
\text { Extremeño de Salud) }\end{array}$ & evalmed.es & España \\
\hline Royal Dutch Society for Physical Therapy (KNGF) & www.fysionet.nl & Holanda \\
\hline American Urogynecologic Society & www.augs.org & EE.UU. \\
\hline CDC's Division of Viral Hepatitis & http://www.cdc.gov/hepatitis/ & EE.UU. \\
\hline
\end{tabular}

CORRESPONDENCIA:

e-mail: kine.rae@gmail.com

\section{BIBLIOGRAFÍA}

1. West S, King V, Carey TS, Lohr KN, McKey N, Sutton SF, et al. Systems to rate strength of scientific evidence. AHRQ 2002; p.47.

2. González de Dios J. Niveles de evidencia y fuerza de las recomendaciones: necesidad de homogenización. Espacio asma 2010;3:24-8.

3. González de Dios J, Ochoa Sangrador C. De la evidencia a la recomendación: una tarea pendiente. Med Clin 2011;135:601-2.

4. Holger JS, Best D, Vist G, Andrew DO, for the GRADE working group. Letters, numbers, symbols and words: how to communicate grades of evidence and recommendations. CMAJ 2003;169:677-80.
5. Atkins D, Best D, Briss PA, Eccles M, Falck-ytter Y, Flottorp $\mathrm{S}$, et al.; Grade working group. Grading quality of evidence and strength of recommendations. BMJ 2004;328:1490-4.

6. Van Tulder M, Furlan A, Bombardier C, Bouter L, Editorial Board of the Cochrane Collaboration Back Review Group. Updated method guidelines for systematic reviews in the cochrane collaboration back review group. Spine 2003;28:1290-9.

7. Martín Muñoz P, González de Dios J. valoración de la calidad de evidencia y fuerza de recomendaciones (I). Evapediatr 2010;6:63.

8. Martín Muñoz P, González de Dios J. Valoración de la calidad de evidencia y fuerza de recomendaciones (II). Consideraciones prácticas en la aplicación del sistema GRADE. Evapediatr 2010;6:91.

9. Nynke S, Van der windt D, Assendelft WJ, Deville WL, Korthals-de bos IB, Bouter LM. Corticosteroid injections, physiotherapy, or a wait-and-see policy for lateral epicondylitis: A randomised controlled trial. Lancet 2002;359:657-62. 